University of New Orleans

ScholarWorks@UNO

2-2001

\title{
Simulations of ice and liquid water over a range of temperatures using the fluctuating charge model
}

Steven W. Rick

University of New Orleans, srick@uno.edu

Follow this and additional works at: https://scholarworks.uno.edu/chem_facpubs

Part of the Chemistry Commons

\section{Recommended Citation}

Steven W. Rick. 2001. "Simulations of ice and liquid water over a range of temperatures using the fluctuating charge model." Journal of Chemical Physics 114 (5): 2276-2283.

This Article is brought to you for free and open access by the Department of Chemistry at ScholarWorks@UNO. It has been accepted for inclusion in Chemistry Faculty Publications by an authorized administrator of ScholarWorks@UNO.For more information, please contact scholarworks@uno.edu. 


\title{
Simulations of ice and liquid water over a range of temperatures using the fluctuating charge model
}

\author{
Steven W. Rick ${ }^{\mathrm{a})}$ \\ Department of Chemistry, University of New Orleans, New Orleans, Louisiana 70148, \\ and Chemistry Department, Southern University of New Orleans, New Orleans, Louisiana 70126
}

(Received 19 April 2000; accepted 7 November 2000)

\begin{abstract}
The temperature dependence of the thermodynamic and dynamical properties of liquid water using the polarizable fluctuating charge (FQ) model is presented. The properties of ice Ih, both for a perfect lattice with no thermal disorder and at a temperature of $273 \mathrm{~K}$, are also presented. In contrast to nonpolarizable models, the FQ model has a density maximum of water near $277 \mathrm{~K}$. For ice, the model has a dipole moment of the perfect lattice of 3.05 Debye, in good agreement with a recent induction model calculation. The simulations at $273 \mathrm{~K}$ and the correct density find that thermal motion decreases the average dipole moment to $2.96 \mathrm{D}$. The liquid state dipole moment is less than the ice value and decreases with temperature. (C) 2001 American Institute of Physics.
\end{abstract}

[DOI: $10.1063 / 1.1336805]$

\section{INTRODUCTION}

For the computer simulation of water and aqueous solutions, there are quite a large number of water potentials, which are used over a wide range of temperature, pressure, and phases. Most potentials are parametrized to be accurate at $25^{\circ} \mathrm{C}$ and $1 \mathrm{~atm} .{ }^{1}$ The charges of the model represent one important part of the parametrization. The nonpolarizable models have a dipole moment in the range of 2.1 to $2.4 \mathrm{D}$, enhanced from the gas phase value of $1.85 \mathrm{D}$. Most polarizable models are constructed to have the correct gas-phase dipole moment and the dipole moment is enhanced in condensed phases by the electric fields from other molecules. The polarizable models are also mostly parametrized to data at $25^{\circ} \mathrm{C}$ and $1 \mathrm{~atm}$, but the hope is that the polarizability of the interactions makes these models more applicable to other thermodynamic states. A third simulation approach, ab initio molecular dynamics, is free from the approximations of a force field. ${ }^{2-4}$ Due to the computational requirements of the $a b$ initio models, their application has so far been limited to small systems and short times, but they have provided a wealth of information about water.

Polarizability is the response of the electronic density to an electric field. In terms of potential models, polarization causes the interactions to be non-pairwise additive. For molecules, the response is mainly of two types: a local change in the density around an atom, and movement of charge density from one atom to another or to a bond. Dipole polarizable models are constructed to treat the first effect. ${ }^{5-19}$ Fluctuating charge models treat the second effect. ${ }^{20-28}$ Another model allows for charge to move from atom to atom as well from atom to bond. ${ }^{29}$ Other models combine both inducible dipoles and fluctuating charges. ${ }^{30,31}$ The fluctuating charge models have a polarization response only along the directions connecting charge sites, generally placed on or near atoms. Therefore, a planar molecule such as water does not

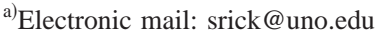

have out-of-plane polarization. For water, the polarization tensor, rather than being nearly isotropic, is then zero for the out-of-plane component and can be overestimated for the direction connecting the hydrogen atoms. ${ }^{21}$ Out-of-plane polarization could be included in fluctuating charge models by adding charge sites which are not in the plane of the molecule. For some properties, it may be that the isotropy of the polarizability response is important. Direct probes of the polarization anisotropy such as the optical Kerr effect spectroscopy and depolarized Raman scattering have been examined computationally. ${ }^{32-34}$ These studies find that the polarizability of liquid water is less isotropic than an isolated water molecule, although this conclusion has been disputed. ${ }^{34}$

A third response of the charge distribution to an electric field is charge transfer between different molecules. The fluctuating charge model can include this response, but in its present version charge transfer is not allowed. ${ }^{21}$ In ab initio calculations of the water dimer, much less charge is transferred between molecules $(0.01 e)$ than is transferred between atoms on the same molecule. ${ }^{35}$ In ab initio molecular dynamics simulations of liquid water, there is also very little charge transfer between molecules, indicating that intermolecular charge transfer may not be important for pure water. ${ }^{4}$ However, some decompositions of quantum-mechanical interaction energies find that charge transfer accounts for a considerable amount of the interaction energy, while others do not. ${ }^{36-39}$ A related question is the covalent nature of the hydrogen bond, which has been suggested in recently measured Compton profile anisotropies of ice. ${ }^{40}$ The anisotropies may not be due to a covalent interaction but to antisymmetrizing of the monomer wave functions, so the importance of covalency and charge transfer on water interactions remains unresolved. ${ }^{41}$

Estimates of the dipole moment of water in the condensed phases vary, since it cannot be directly measured. An induction model calculation for the ice Ih lattice gives a dipole moment of $3.09 \mathrm{D}^{42}$ This calculation used more accu- 
rate data as input as well as higher order terms in the multipolar expansion than the 1966 calculation of Coulson and Eisenberg, which gave $2.6 \mathrm{D}^{43}$ Density functional theory calculations on the ice Ih lattice find that the dipole moment can vary between 2.33 and 2.97 depending on how the electronic density is assigned to molecules. ${ }^{44,45}$ The ab initio simulations of liquid water, at $25^{\circ} \mathrm{C}$ and $1 \mathrm{~atm}$, give three different average dipole moments: $2.66,{ }^{2} 2.43,{ }^{4}$ and $2.95 \mathrm{D} .{ }^{3}$ Each of the three simulations uses a different method for assigning the electronic density to molecules. The choice of a partitioning method clearly changes the value of the dipole moment. The atoms in molecules method (used in Refs. 4 and 42) tends to give smaller dipole moments than the Voronoi method (used in Refs. 2 and 42). These studies find quadrupole moments which are enhanced from the gas-phase values as well. ${ }^{3,4,42}$ The static dielectric constant provides another estimate of the dipole moment of a water molecule. Water models with dipole moments ranging from 2.4 to 2.7 D have a dielectric constant near 80 , at $25^{\circ} \mathrm{C}$ and 1 atm..$^{1,46,47}$ All of these estimates point to a dipole moment of water in the range of 2.4 to $3.0 \mathrm{D}$, at standard temperature and pressure. As significant as the enhancement of the moments is the broad distribution of values the dipole moment takes in liquid water. A broad distribution of dipole moments in seen in both the $a b$ initio and simulations with polarizable potentials. ${ }^{3,4,16,21,25}$ The fluctuations in the electronic distributions are important to the dynamics of water. The Watanabe-Klein model of water, which has a fixed dipole moment of $2.6 \mathrm{D}$, gives accurate static properties but the dynamical properties are about a factor of 2 too slow. ${ }^{48}$ Polarizable models with an average dipole of $2.6 \mathrm{D}$ are accurate for both equilibrium and dynamical properties, indicating that the polarization fluctuations are coupled to transport properties. ${ }^{21,47,49}$ The coupling of translational motion and the dipole moment is evident experimentally as well. The far infrared ${ }^{50}$ and depolarized Raman ${ }^{51}$ spectra have a band near $200 \mathrm{~cm}^{-1}$ that is present in the calculated spectra only if polarization effects are included. ${ }^{21,24,52-54}$ This feature corresponds to translational vibration in the cage of a molecule's nearest neighbors. ${ }^{55}$ This motion only shows up in the dielectric spectrum if it causes a change in the molecule's dipole moment magnitude, since it does not change its orientation.

The importance of polarizability is also demonstrated in comparisons between the FQ model and electronic structure data. ${ }^{26,56}$ In the study of Liu et al., the energies of 57 trimers randomly selected from liquid state simulations plus some selected for a specific geometry were compared using electronic structure methods (Hartree-Fock with 6-31G** basis sets and local Møller-Plesset second-order perturbation theory) and the fluctuating data TIP4P-FQ potential. ${ }^{21}$ For the strength of the three-body interactions, which are up to 1 $\mathrm{kcal} / \mathrm{mol}$ or $10 \%$ of the trimer binding energy, the FQ model agrees as well with the electronic structure methods. If charge transfer is introduced into the FQ model the agreement becomes much worse, even after the potential is reparametrized by fitting to the $a b$ initio three-body energies. ${ }^{26}$ In the study of Wood et al., perturbation theory was used to calculate the free energy of $a b$ initio water, using a classical potential (TIP4P and TIP4P-FQ) as a reference. The
TIP4P-FQ model was found to predict the interaction energies well, in fact much better than the TIP4P model. These two studies imply that the FQ model gives accurate values for the energies of individual configurations. Ranking different configurations correctly is important to describing water at lower temperatures since different lower energy structures begin to predominate.

The temperature dependence of water properties has been examined for many water potentials. Most nonpolarizable models do demonstrate the well-known density maximum of water, but not near the the experimental value of 277 K. ${ }^{24,57-61,62-65}$ The TIP4P ${ }^{66}$ and SPC/E ${ }^{67}$ models, two of the most commonly used water pair potentials, have a temperature of maximum density, $T_{\mathrm{MD}}$, near $248 \mathrm{~K}^{60,62,64}$ and the ST2 model has a $T_{\mathrm{MD}}$ near $320 \mathrm{~K}^{58,62}$ The polarizable point charge (PPC) model has been shown to have a $T_{\mathrm{MD}}$ right at $277 \mathrm{~K}^{24}$ and variations of the TIP4P-FQ model in which the Lennard-Jones size parameter $\sigma$ is coupled to the oxygen charge value exhibit a $T_{\mathrm{MD}}$ near $277 \mathrm{~K} .{ }^{28}$ Other polarizable models, using point inducible dipoles, are not an improvement over nonpolarizable models. The nonempirical molecular orbital (NEMO) model does not demonstrate a density maximum and the Brodholt, Sampoli, nd Vallauri (BSV) model has a density maximum at the right temperature but the density is much too high and the temperature dependence of the density is much too strong. ${ }^{61,65}$ Closely related to the density maximum is the freezing point. The freezing points which have been calculated (for TIP4P and SPC/E) are too low: TIP4P freezes at $214 \mathrm{~K}$ and SPC/E freezes at $200 \mathrm{~K}^{68,69}$ It should be noted that some of these studies (including both freezing point calculations ${ }^{68,69}$ and several of the $T_{\mathrm{MD}}$ calculations $^{60,61,64,65}$ ) were done without treating the longranged electrostatic interactions with Ewald sums. The use of Ewald sums has been shown to be particularly important for simulating polarizable systems and is important for nonpolarizable potentials as well. ${ }^{70,71}$ Using Ewald sums the results for the melting temperature and $T_{\mathrm{MD}}$ may be closer to the experimental values. For the SPC/E potential, the simulations with Ewald sums find a $T_{\mathrm{MD}}$ about 10 deg closer to the experimental value than those without Ewald sums. ${ }^{60,62,63}$ The polarizable BSV potential designed for the ice Ih phase does not represent the properties of liquid water well and so apparently no one potential has been shown to accurately reproduce the properties of both the liquid and the ice phase. ${ }^{72}$ Accuracy in the description of ice is important for liquid water models because the presence of solutes perturbs the solvent to become more structured. Confidence in the predictions of solvent structure around solutes will therefore be increased if the potential has been demonstrated to be a good model for ice. For gas-liquid coexistence, which has been studied in more depth than ice-liquid coexistence, it has been shown that, in general, polarizable models, including TIP4P-FQ, do no better than nonpolarizable models in predicting the critical point. ${ }^{73-76}$ However, Kiyohara et al. showed small changes in the potential parameters of a polarizable model lead to large changes in the liquid-gas coexistence properties. ${ }^{73}$

The TIP4P-FQ model has been shown to represent liquid water at $298 \mathrm{~K}$ and $1 \mathrm{~atm}$ well, giving fairly accurate values 
for the energy, pressure, diffusion constant, the dielectric constant (and also the frequency-dependent dielectric constant), and the pair correlation function. ${ }^{21}$ The model was found to reproduce the experimental neutron scattering data on pure water. ${ }^{77}$ The potential has been successfully applied to many systems, including aqueous solutions, ${ }^{22,78,79}$ electronic transitions in aqueous solutions ${ }^{80}$ water on salt surfaces, ${ }^{81}$ gas-liquid coexistence, ${ }^{74,76,82}$ and quantummechanical/molecular mechanics (QM/MM) calculations. ${ }^{25,83,84}$ In this study, the properties of the TIP4P-FQ model at temperatures below $298 \mathrm{~K}$ for liquid water and ice will be examined. In addition, the sensitivity of the solid state properties on the Lennard-Jones parameter $\sigma$ will be presented.

\section{METHODS}

The fluctuating charge model is a polarizable potential model in which the partial charges on atomic sites are treated as variables which respond to changes in their environments. ${ }^{21}$ The model uses the concept of electronegativity equalization. ${ }^{85-91}$ The TIP4P-FQ model uses the geometry of the TIP4P water model and includes Lennard-Jones interactions between oxygen sites and three charge sites: two on the hydrogen atoms and one on the M site $0.15 \AA$ from the oxygen atom. ${ }^{66}$ The FQ model has additional interactions between charge sites on the same molecule. The charges are found by minimizing the energy subject to a charge neutrality constraint. Rather than solving for the charges exactly at each time step, the method treats them as dynamical variables, which are propagated in an extended Lagrangian formalism at a low temperature so as to remain near the potential energy minimum. An advantage of the fluctuating charge model is that it introduces no new interactions and using the extended Lagrangian technique for updating the charges makes the model only about $10 \%$ more computationally expensive than nonpolarizable models. ${ }^{21}$ Dipole polarizable models are about a factor of 2 to 4 times more expensive. ${ }^{9,92}$

The simulations to calculate the density as a function of temperature were done in the isothermal-isobaric (constant $\mathrm{T}, \mathrm{P}, \mathrm{N}$ ) ensemble, by coupling to a pressure bath (at $1 \mathrm{~atm}$ ) and a Nosé-Hoover temperature bath. ${ }^{93-97}$ For temperatures at $290 \mathrm{~K}$ and below, the simulations were run for $2.5 \mathrm{~ns}$ and at higher temperatures the simulations were run for $1.0 \mathrm{~ns}$. To calculate the dielectric constant, the simulations were run for $5 \mathrm{~ns}$ simulations in the canonical (constant $\mathrm{T}, \mathrm{V}, \mathrm{N}$ ) ensemble, setting the density equal to the average density from the constant pressure simulations. The simulations to calculate the diffusion constant (from the Einstein relation) were done in the microcanonical $(\mathrm{E}, \mathrm{V}, \mathrm{N})$ ensemble and comprised ten simulations of $100 \mathrm{ps}$ each. The simulations of ice were run for $4 \mathrm{~ns}$ and done in the isothermal-isobaric ensemble with an orthorhombic box with the lengths of each box side treated as an independent variable. ${ }^{98}$ The Nosé variable mass

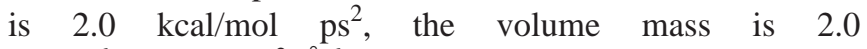
$\times 10^{-6} \mathrm{kcal} / \mathrm{mol} \mathrm{ps}^{2} / \AA^{6}$, the length mass (for the ice simulations) is $10.0 \mathrm{kcal} / \mathrm{mol} \mathrm{ps} / \AA^{2}$, and the charge mass is 6.0 $\times 10^{-5} \mathrm{kcal} / \mathrm{mol} \mathrm{ps}^{2} / \mathrm{e}^{2}$. At the beginning of each trajectory and at $10 \mathrm{ps}$ intervals the exact set of minimum energy charges is found. The liquid simulations used 256 molecules.

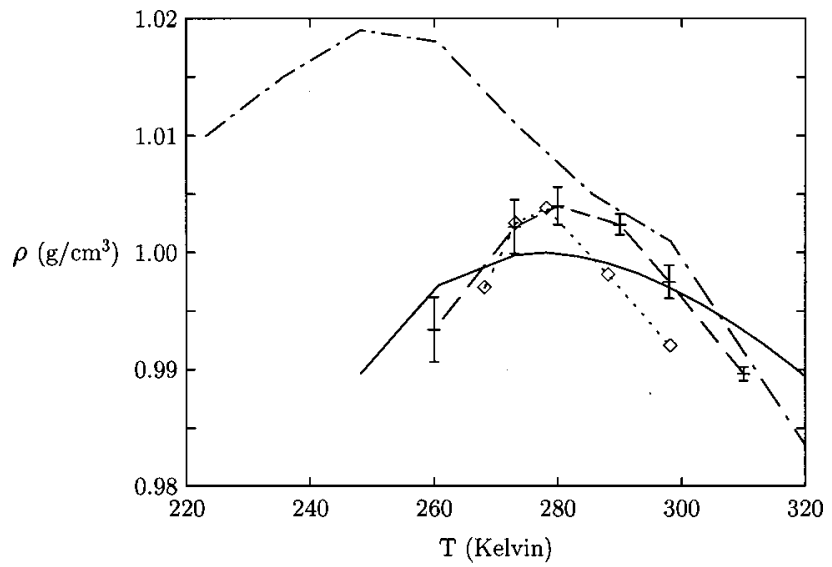

FIG. 1. The density as a function of temperature at $P=1 \mathrm{~atm}$. Simulation results for the TIP4P-FQ model (dashed line and crosses with error bars), the TIP4P model (Ref. 64) (dotted-dashed line) and the PPC model (Ref. 24) (dotted line and diamonds) are compared to the experimental data (Ref. 101) (solid line).

The ice simulations began with a $5 \times 3 \times 3$ orthorhombic unit cell from Hayward and Reimers which contained 360 molecules. ${ }^{99}$ This unit cell was generated with the oxygen atoms organized on a lattice and the hydrogen atoms disordered in such a way that the net dipole and quadrupole moments of the unit cell are zero. All simulations used the Ewald method for treating long-ranged electrostatic interactions with the screening parameter equal to $5 / \mathrm{L}$, where $\mathrm{L}$ is the simulation box side length, 256 lattice vectors in the Fourier space sum and conducting boundary conditions. ${ }^{100}$

\section{RESULTS}

\section{A. Density}

The liquid density as a function of temperature for the TIP4P-FQ model is shown in Fig. 1, along with the experimental density and the results for the TIP4P potential and the PPC potential. ${ }^{24,64,101}$ The error bars represent $95 \%$ confidence intervals. The TIP4P-FQ model exhibits a temperature of maximum density, $T_{\mathrm{MD}}$, at $280 \mathrm{~K}$, near the experimental value, and the improvement over the nonpolarizable TIP4P model is significant. The results are similar to the results for the PPC model, with some differences around $300 \mathrm{~K}$. The PPC and TIP4P-FQ models are similar; both have charges which respond to the electric field of the environment and both use an $\mathrm{M}$ site for the negative charge. However, the details of the charge response to the electric field are different; the geometries (both rigid) are different and in the PPC model the position of the $\mathrm{M}$ site can move in the plane of the molecule. Also, the zero field dipole moment of the PPC model is $2.14 \mathrm{D}$, not $1.85 \mathrm{D}$ as it is for the TIP4P-FQ model. The TIP4P-FQ density results are much more curved than the experimental data and this is true of all the computed densities near the density maximum..$^{24,28,59-61,64,65}$ Below 260 $\mathrm{K}$, the liquid forms a glass, as has been found in another simulation of water at temperatures below $T_{\mathrm{MD}} \cdot{ }^{60}$ The glassy state is indicated by discontinuous decreases in the heat capacity and isothermal compressibility. 
TABLE I. Properties of the TIP4P-FQ model for the liquid and solid phase: density $(\rho)$, average dipole moment $(\langle|\mu|\rangle)$, the root-mean-square of the dipole moment distribution $\left(\left\langle\delta \mu^{2}\right\rangle^{1 / 2}\right)$, the static dielectric constant $\left(\epsilon_{0}\right)$, the translational diffusion constant $(D)$, the NMR relaxation time $\left(\tau_{\mathrm{NMR}}\right)$, the heat of vaporization, or sublimation for the solid $\left(\Delta H_{\text {vap }}\right)$, and the isothermal compressibility $(\kappa)$. Also shown are the experimental values, which for some temperatures are interpolations between the reported data points.

\begin{tabular}{|c|c|c|c|c|c|c|c|c|c|}
\hline $\begin{array}{c}T \\
(\mathrm{~K})\end{array}$ & & $\begin{array}{c}\rho \\
\left(\mathrm{g} / \mathrm{cm}^{3}\right)\end{array}$ & $\begin{array}{l}\langle|\mu|\rangle \\
\text { (D) }\end{array}$ & $\begin{array}{c}\left\langle\delta \mu^{2}\right\rangle^{1 / 2} \\
\text { (D) }\end{array}$ & $\epsilon_{0}$ & $\begin{array}{c}D \\
\left(10^{-9} \mathrm{~m}^{2} / \mathrm{s}\right)\end{array}$ & $\begin{array}{l}\tau_{\mathrm{NMR}} \\
(\mathrm{ps})\end{array}$ & $\underset{(\mathrm{kcal} / \mathrm{mol})}{\Delta H_{\mathrm{vap}}}$ & $\begin{array}{c}\kappa \\
\left(10^{-6} \mathrm{bar}^{-1}\right)\end{array}$ \\
\hline \multicolumn{10}{|l|}{$\overline{\text { Liquid }}$} \\
\hline \multirow[t]{2}{*}{260} & calc. & $0.993(3)$ & $2.805(5)$ & $0.208(2)$ & $105(27)$ & $0.19(1)$ & $20(2)$ & $11.60(3)$ & $31(3)$ \\
\hline & exp. & $0.9970^{\mathrm{a}}$ & & & & $0.64^{\mathrm{b}}$ & & $10.90^{\mathrm{c}}$ & $58.0^{\mathrm{d}}$ \\
\hline \multirow[t]{2}{*}{273} & calc. & $1.002(2)$ & $2.733(4)$ & $0.206(1)$ & $97(19)$ & $0.67(7)$ & $8(2)$ & $11.15(2)$ & $37(4)$ \\
\hline & exp. & $0.9998^{\mathrm{a}}$ & & & $87.9(2)^{\mathrm{e}}$ & $1.09^{\mathrm{b}}$ & & $10.76^{\mathrm{c}}$ & $50.9^{\mathrm{d}}$ \\
\hline \multirow[t]{2}{*}{280} & calc. & $1.004(2)$ & $2.702(3)$ & $0.205(2)$ & $93(15)$ & $0.91(5)$ & $4.2(4)$ & $10.95(2)$ & $37(2)$ \\
\hline & exp. & $0.9999^{\mathrm{a}}$ & & & $84.7(2)^{\mathrm{e}}$ & $1.38^{\mathrm{b}}$ & & $10.69^{\mathrm{c}}$ & $48.6^{\mathrm{d}}$ \\
\hline \multirow[t]{2}{*}{290} & calc. & $1.002(1)$ & $2.665(1)$ & $0.203(1)$ & $88(4)$ & $1.44(9)$ & $2.8(1)$ & $10.69(1)$ & $38(2)$ \\
\hline & exp. & $0.9988^{\mathrm{a}}$ & & & $81.3(1)^{\mathrm{e}}$ & $1.86^{\mathrm{b}}$ & $3.08^{\mathrm{f}}$ & $10.59^{\mathrm{c}}$ & $46.4^{d}$ \\
\hline \multirow[t]{2}{*}{298} & calc. & $0.998(1)$ & $2.641(1)$ & $0.201(1)$ & $79(8)$ & $1.93(9)$ & $2.1(1)$ & $10.51(1)$ & $38(2)$ \\
\hline & exp. & $0.9970^{\mathrm{a}}$ & & & $78.36(5)^{\mathrm{e}}$ & $2.30^{\mathrm{b}}$ & $2.46^{\mathrm{f}}$ & $10.51^{\mathrm{c}}$ & $45.2^{\mathrm{d}}$ \\
\hline \multirow[t]{2}{*}{310} & calc. & $0.990(1)$ & $2.606(2)$ & $0.200(2)$ & $78(2)$ & $2.66(9)$ & $1.4(1)$ & $10.25(1)$ & $40(2)$ \\
\hline & exp. & $0.9933^{\mathrm{a}}$ & & & $74.2(1)^{\mathrm{e}}$ & $3.04^{\mathrm{b}}$ & $1.93^{\mathrm{f}}$ & $10.39^{\mathrm{c}}$ & $44.3^{\mathrm{d}}$ \\
\hline \multicolumn{10}{|l|}{ Ice } \\
\hline \multirow[t]{2}{*}{273} & calc. & $0.968(1)$ & $3.097(1)$ & $0.152(1)$ & & & & $13.39(1)$ & $13(4)$ \\
\hline & exp. & $0.920^{\mathrm{g}}$ & & & & & & $12.20^{\mathrm{g}}$ & \\
\hline
\end{tabular}

${ }^{\mathrm{a}}$ Reference 101.

${ }^{\mathrm{b}}$ Reference 113 .

${ }^{\mathrm{c}}$ Reference 117.

${ }^{\mathrm{d}}$ Reference 107.
${ }^{\mathrm{e}}$ Reference 106.

${ }^{\mathrm{f}}$ Reference 116.

${ }^{g}$ Reference 102.
The density for ice at $273 \mathrm{~K}$ is $0.968 \mathrm{~g} / \mathrm{cm}^{3}$, less than the density of the liquid at the same temperature (see Table I). This is greater than the experimental density of ice Ih at 273 $\mathrm{K}$, which is $0.92 \mathrm{~g} / \mathrm{cm}^{3} .{ }^{102}$ The ice density of other models is also greater than the experimental value. The TIP4P model gives a density of $0.963 \mathrm{~g} / \mathrm{cm}^{3}$ at $214 \mathrm{~K}$ and 0.944 at $270 \mathrm{~K}$, and the SPC/E model gives a density of 0.951 at 200 K. ${ }^{68,69,103}$ Other polarizable models give densities which are less than the experimental value, at $0 \mathrm{~K} .{ }^{11,14}$ The lattice constants are $\mathrm{a}=4.43 \AA$ and $\mathrm{c}=7.27 \AA$, giving a c/a ratio which is greater than that of a perfect tetrahedron (1.633) rather than less, as is observed in ice Ih crystals. ${ }^{102}$ The ice configurations after $4 \mathrm{~ns}$ of simulation at $273 \mathrm{~K}$ were minimized. The resulting structure, by visual inspection, demonstrated no lattice defects and still retained the disordered hydrogen arrangement of the initial lattice.

\section{B. Dipole and quadrupole moments}

The average dipole moments for liquid water and ice are given in Table I. Also shown is the square root of the variance of the dipole moment distribution, $\left\langle\delta \mu^{2}\right\rangle^{1 / 2}$. For the liquid, the dipole moment steadily decreases as a function of temperature and the distribution gets narrower. Widths for the dipole moment distributions can be calculated from the data reported in the study of Jedlovszky and Vallauri for three different dipole polarizable models and those values, at $298 \mathrm{~K}$, are all near $0.2 \mathrm{D}$, close to the value for the TIP4P-FQ model. ${ }^{65}$ The width of the dipole moment from an $a b$ initio study of liquid water, at $298 \mathrm{~K}$, is larger, $0.35 \mathrm{D}^{4}$ The TIP4P-FQ dipole moment in the solid phase is larger than the liquid and the distribution is more narrow, indicating a more homogeneous environment. One other study using a polarizable potential and one $a b$ initio study also found a larger dipole moment for ice relative to the liquid, and this dipole moment for ice agrees with other estimates. ${ }^{4,42,44,45,72}$ The larger dipole moment for ice is also consistent with the larger dielectric constant of ice. ${ }^{102}$

The dipole and quadrupole moments of the TIP4P-FQ model for various state points are shown in Table II, along with the values from other studies. As stated previously, the value of the condensed-phase multipole moments calculated from $a b$ initio methods depends on how the electronic density is partitioned. The perfect lattice results are for a lattice with a density near $0.92 \mathrm{~g} / \mathrm{cm}^{3}$ with no thermal disorder. The

TABLE II. Average values of the dipole and quadrupole moments of water.

\begin{tabular}{lcccc}
\hline \hline & $\mu$ & $Q_{x x}$ & $Q_{y y}$ & $Q_{z z}$ \\
& $(\mathrm{D})$ & $(\mathrm{D}) \AA$ & $(\mathrm{D}) \AA$ & $(\mathrm{D}) \AA$ \\
\hline Gas phase & & & & \\
TIP4P-FQ & 1.85 & -1.79 & 1.88 & -0.09 \\
Experiment & $1.855^{\mathrm{a}}$ & $-2.50^{\mathrm{b}}$ & $2.63^{\mathrm{b}}$ & $-0.13^{\mathrm{b}}$ \\
& & & & \\
Liquid & 2.73 & -2.60 & 2.74 & -0.14 \\
TIP4P-FQ $(T=273 \mathrm{~K})$ & 2.64 & -2.51 & 2.64 & -0.13 \\
TIP4P-FQ $(T=298 \mathrm{~K})$ & $2.95^{\mathrm{c}}$ & $-3.16^{\mathrm{c}}$ & $3.38^{\mathrm{c}}$ & $-0.22^{\mathrm{c}}$ \\
ab initio $(T=298 \mathrm{~K})$ & $2.43^{\mathrm{d}}$ & $-2.67^{\mathrm{d}}$ & $2.77^{\mathrm{d}}$ & $-0.10^{\mathrm{d}}$ \\
ab initio $(T=298 \mathrm{~K})$ & & & & \\
& & & & \\
Ice & 3.10 & -2.96 & 3.12 & -0.16 \\
TIP4P-FQ $(T=273 \mathrm{~K})$ & 3.05 & -2.93 & 3.08 & -0.15 \\
TIP4P-FQ (perfect lattice) & $3.09^{\mathrm{e}}$ & $-3.14^{\mathrm{e}}$ & $3.29^{\mathrm{e}}$ & $-0.15^{\mathrm{e}}$ \\
Induction model (perfect lattice) & $2.67^{\mathrm{d}}$ & $-2.76^{\mathrm{d}}$ & $2.84^{\mathrm{d}}$ & $-0.08^{\mathrm{d}}$ \\
ab initio (perfect lattice) & & & & \\
\hline
\end{tabular}

${ }^{\mathrm{a}}$ Reference 104. $\quad{ }^{\mathrm{d}}$ Reference 4.

${ }^{\mathrm{b}}$ Reference 105 .

${ }^{\mathrm{c}}$ Reference 3. 


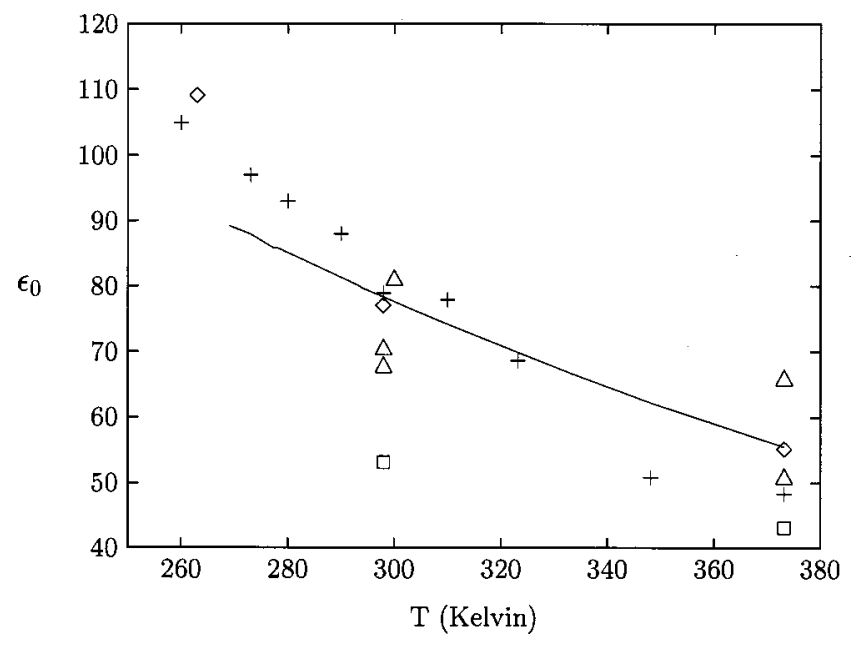

FIG. 2. The static dielectric constant, $\epsilon_{0}$, comparing simulation results for the TIP4P-FQ model (crosses, the value at $298 \mathrm{~K}$ is from Ref. 21 and the values at 323,348 , and $373 \mathrm{~K}$ are from Ref. 82), the TIP4P model (Ref. 121) (squares), the SPC/E model (Refs. 108-110) (triangles), the PPC model (Ref. 24) (diamonds), and the experimental data (Refs. 106, 107) (solid line)

ice results at $273 \mathrm{~K}$ are averages over the 4 nanosecond simulation. The origin for the quadrupole moments is the center of mass except for the results of Ref. 4, which use the oxygen position. The $x$ direction is out of the molecular plane, the $y$ direction connects the two hydrogen atoms, and the $z$ direction is along the $\mathrm{C}_{2}$ axis away from the oxygen atom. In general, the quadrupole moments are enhanced for the condensed phases, just like the dipole moments. The moments for the TIP4P-FQ model for the perfect ice lattice are very close to induction model results for a very similar lattice (see Table II). ${ }^{42}$

\section{Dielectric constant}

The dielectric constant, $\epsilon$, was calculated from

$$
\epsilon=\epsilon_{\infty}+\left(\frac{4 \pi \rho}{3 k T}\right)\left(\frac{\left\langle\mathbf{M}^{2}\right\rangle}{N_{\text {molec }}}\right),
$$

where $\rho$ is the density, $k$ is Boltzmann's constant, $\left\langle\mathbf{M}^{2}\right\rangle$ is the average of the square of the total dipole moment of the central simulation box, and $N_{\text {molec }}$ is the total number of molecules. ${ }^{100}$ The values are shown in Fig. 2 and Table I. Also plotted are the experimental values ${ }^{106,107}$ and the results for other potentials. The TIP4P-FQ model provides a good estimate for the dipole moment over a range of temperatures. The value at $298 \mathrm{~K}$ is from Ref. 21 and the high temperature values are from Medeiros and Costas. ${ }^{82}$ The TIP4P-FQ model may be overestimating the temperature dependence, although the error bars are large (see Table I). The error estimates for the high temperature dielectric constant are large also, about $\pm 40 .{ }^{82}$ The PPC model also gives good values for the dielectric constant over a range of temperatures. ${ }^{24}$ The SPC/E model appears to be accurate as well, although different studies find a range of values. ${ }^{108-110}$ For a comparison of the dielectric constants of many water models at $298 \mathrm{~K}$, see Refs. 1 and 44.

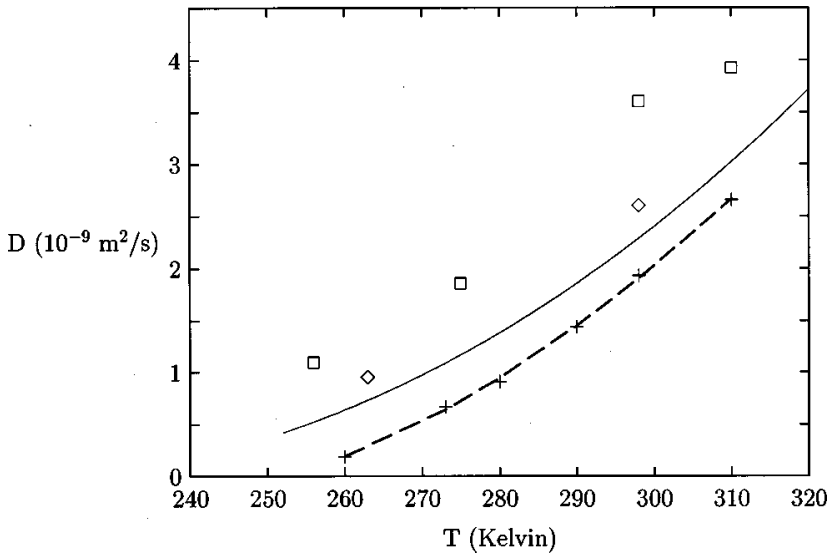

FIG. 3. The diffusion constant for water, comparing simulation results for the TIP4P-FQ model (crosses and dashed line fit), the TIP4P model (Refs. $48,111)$ (squares), the PPC model (Ref. 24) (diamonds), and the experimental data (Refs. 112, 113) (solid line).

\section{Dynamical properties}

Figure 3 shows the diffusion constant for the TIP4P-FQ, PPC, and TIP4P models along with the experimental values. $^{24,48,111-113}$ The TIP4P-FQ results slightly underestimate the diffusion constant over the temperature range. The fit to the results is made to the form $D=D_{0} T^{1 / 2}\left(T / T_{S}\right.$ $-1)^{\gamma}$, which has been shown empirically to fit the isobaric temperature dependence of transport properties of water. $^{113,114}$ The fitting parameters are $D_{0}=1.15$ $\times 10^{-9} \mathrm{~m}^{2} / \mathrm{s}, T_{S}=250 \mathrm{~K}$, and $\gamma=1.42$, which can be compared to the experimental values of $D_{0}=0.87 \times 10^{-9} \mathrm{~m}^{2} / \mathrm{s}$, $T_{S}=220 \mathrm{~K}$, and $\gamma=1.81 .{ }^{113}$ The exponent $\gamma$, which gives the temperature dependence of the diffusion constant, is close to the experimental value. The exponent for the SPC/E model is 2.8..$^{115}$

Other dynamical information is contained in rotational times. Rotations about different molecular axes may be sensitive to the anisotropies of the potential. As mentioned in the Introduction, the TIP4P-FQ model does not have a nearly isotropic polarizability tensor. Taking the plane of the molecule to be the $z y$ plane, the dipole $\left(\mathrm{C}_{2}\right)$ axis along $z$ and the direction connecting the hydrogen atoms as $y$, then the polarizability tensor, rather than being about $1.5 \AA$ for all diagonal components, is $\left(\alpha_{x x}, \alpha_{y y}, \alpha_{z z}\right)=(0,2.55 \AA, 0.82 \AA) .{ }^{21}$ The out-of-plane component, $\alpha_{x x}$, is zero. The experimentally accessible rotational time constant is for rotations of a molecule about its $y$ axis, which can be measured using nuclear magnetic resonance (NMR). Figure 4 shows this relaxation time, $\tau_{\mathrm{NMR}}$, for the TIP4P-FQ model, the TIP4P model, ${ }^{48}$ and from experiment. ${ }^{116}$ The values of $\tau_{\mathrm{NMR}}$ are also given in Table I. Over the range of the experimental data, over $10^{\circ} \mathrm{C}$, the agreement between experiment and the TIP4P-FQ model is good. For this rotation, and for translational diffusion as well, the lack of out-of-plane polarizability does not appear to lead to large errors. Rotational time scales are also given by the Debye relaxation time, $\tau_{D}$, which gives the time scale for rotations of the dipole moment of the entire system. For the TIP4P-FQ model, this has been shown to be in good agreement with the experimental value at $298 \mathrm{~K}^{21}$ The TIP4P model also gives an accurate $\tau_{D}{ }^{48}$ so 


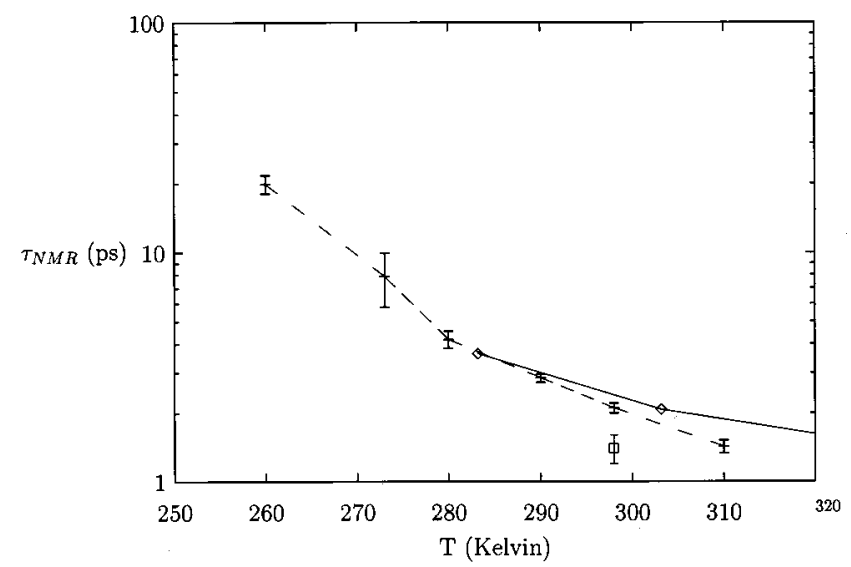

FIG. 4. The rotational time constant for water, $\tau_{\mathrm{NMR}}$, comparing simulation results for the TIP4P-FQ model (crosses and dashed line) and the TIP4P model (Ref. 48) (square) and the experimental values (Ref. 116) (solid line). Shown on a semilog scale.

this may not be as sensitive to the treatment of polarizability as $D$ or $\tau_{\mathrm{NMR}}$.

\section{E. Heats of vaporization and sublimation}

The heats of vaporization and sublimation are found from

$$
\Delta H_{\mathrm{vap}}=-\langle E(\text { liquid })\rangle / N+R T,
$$

and

$$
\Delta H_{\text {sub }}=-\langle E(\text { solid })\rangle / N+R T,
$$

where $E$ (liquid) is the energy of the liquid, $E$ (solid) is the energy of the solid, and $N$ is the number of molecules. ${ }^{64}$ The results for the TIP4P-FQ model are given in Table I and plotted in Fig. 5, along with the experimental data and the TIP4P values. ${ }^{64,102,117}$ The TIP4P-FQ model overestimates both $\Delta H_{\text {vap }}$ at low temperatures and $\Delta H_{\text {sub }}$, indicating that the energy is too attractive. The lattice energy for the perfect lattice with a density of $0.92 \mathrm{~g} / \mathrm{cm}^{3}$ is $-14.26 \mathrm{kcal} / \mathrm{mol}$, in close agreement with the experimental lattice energy of

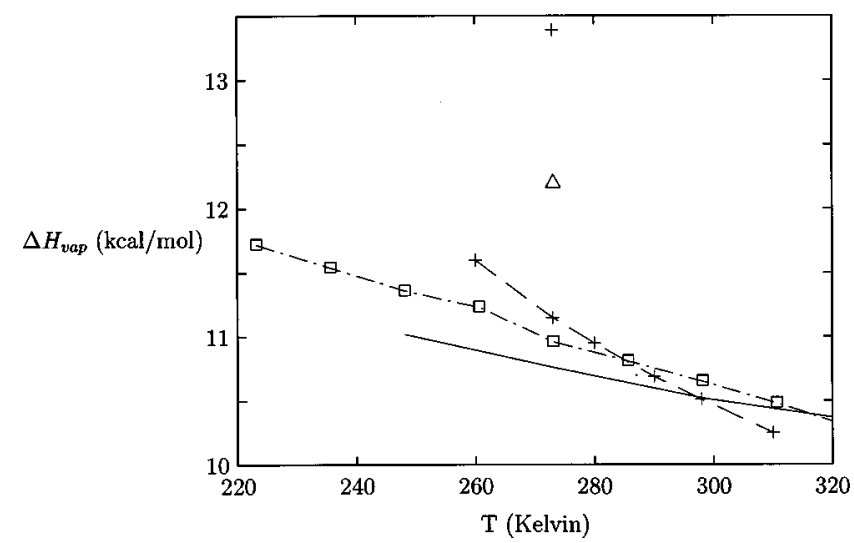

FIG. 5. The heat of vaporization for the TIP4P-FQ model (dashed line and crosses), the TIP4P model (Ref. 64) (dotted-dashed line and squares), and the experimental results (Ref. 117) (solid line) and the heat of sublimation for the TIP4P-FQ model (crosses) and the experimental value (Ref. 102) (triangle).

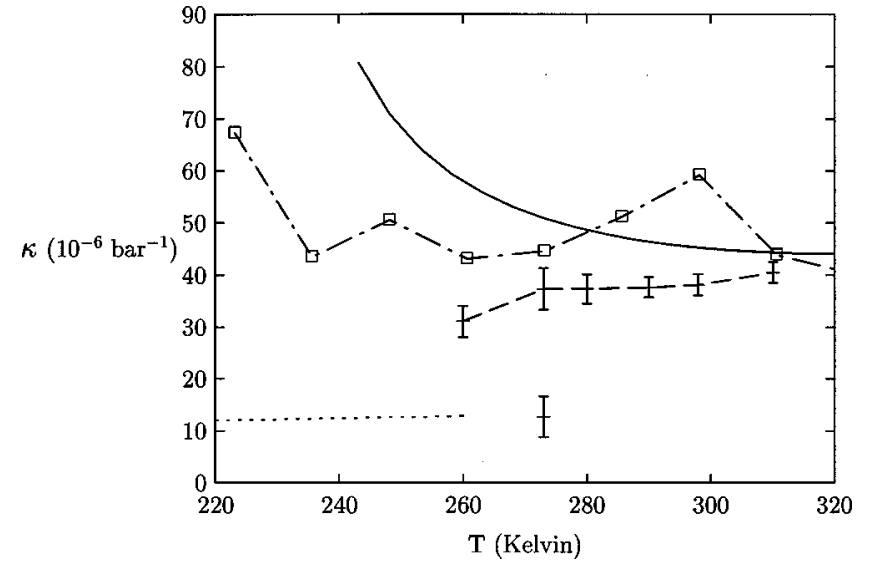

FIG. 6. The isothermal compressibility for water and ice. Simulation results for the TIP4P-FQ model (crosses with error bars and the dashed line for the liquid values), TIP4P model for the liquid (Ref. 64) (dotted-dashed line and squares), and the experimental results for water (Ref. 107) (solid line) and ice (Ref. 102) (dotted line).

$-14.08 \mathrm{kcal} / \mathrm{mol} .^{118}$ Thus, the dipole moment and the energy of the perfect lattice are accurately represented by the TIP4P-FQ model. However, when the lattice is allowed to relax it becomes too dense and too low in energy, perhaps, since the dipole and quadrupole moments seem accurate, due to errors from the Lennard-Jones functional form. Alternatively, the errors in the energy and density may be from the increased anisotropy of the polarizability. It follows from the large slope of $\Delta H_{\text {vap }}$ that the heat capacity for the liquid is larger than the experimental values as well.

\section{F. Isothermal compressibility}

The isothermal compressibility can be calculated from ${ }^{100}$

$$
\kappa=-\frac{1}{V}\left(\frac{\partial V}{\partial P}\right)_{N, T}=\frac{1}{k T\langle V\rangle_{N, P, T}}\left(\left\langle V^{2}\right\rangle_{N, P, T}-\langle V\rangle_{N, P, T}^{2}\right) .
$$

The results for the TIP4P-FQ model for the liquid and solid phases are shown in Fig. 6, along with the results for the TIP4P liquid and the experimental data. ${ }^{64,102,107}$ The TIP4P-FQ results appear to have the opposite temperature dependence of the experimental results and the liquid gets less, rather than more, compressible as the temperature decreases towards the freezing point. The TIP4P-FQ model gives a good estimate for the compressibility for the solid.

\section{G. The Lennard-Jones parameter $\sigma$ and solid properties}

Since the solid density of the ice phase as given by the TIP4P-FQ model is too large, a simple improvement can be made by increasing the $\sigma$ parameter of the Lennard-Jones interaction, $\mathrm{U}_{\mathrm{LJ}}$, between oxygen atoms $\left[\mathrm{U}_{\mathrm{LJ}}(\mathrm{r})\right.$ $\left.=4 \epsilon\left[(\sigma / r)^{12}-(\sigma / r)^{6}\right]\right]$. Adjusting $\sigma$ by only $0.4 \%$ from 3.159 to $3.173 \AA$ gives a solid density of $0.921 \mathrm{~g} / \mathrm{cm}^{3}$. For the perfect lattice, the modified potential has a lattice energy of $-13.80 \mathrm{kcal} / \mathrm{mol}$, increased from the TIP4P-FQ lattice energy, and a dipole moment which is unchanged since the electrostatic parts of the potential are the same. With this 
choice of $\sigma$, simulations of ice at $273 \mathrm{~K}$ were run for $1 \mathrm{~ns}$. The average dipole moment is $2.96 \mathrm{D}$, decreased by over 0.1 $\mathrm{D}$ from the TIP4P-FQ value, and less than the dipole moment of the perfect lattice, so that thermal disorder at $273 \mathrm{~K}$ and at the proper density decreases the dipole moment. The value of $\left\langle\delta \mu^{2}\right\rangle^{1 / 2}$ decreases from $0.152 \mathrm{D}$ to $0.135 \mathrm{D}$. The heat of sublimation decreases to $12.56 \mathrm{kcal} / \mathrm{mol}$, in better agreement with the experimental value of $12.2 \mathrm{kcal} / \mathrm{mol}^{102}$ and the solid becomes slightly less compressible, with an isothermal compressibility equal to $10 \times 10^{-6} \mathrm{bar}^{-1}$.

\section{DISCUSSION}

One way to judge the importance of specific effects (polarizability, charge transfer, covalent hydrogen bonds, quantized hydrogens, flexibility, off-atom interaction sites) is to examine if there are experimental properties which can be reproduced only if these effects are included. As discussed in the Introduction, the polarization response is coupled to the value of both the static dielectric constant and the translational diffusion constant. The TIP4P-FQ model accurately reproduces the temperature of maximum density, $T_{\mathrm{MD}}$, which suggests that polarizable interaction energies may be important for this property as well (see Fig. 1). However, since the TIP4P and SPC/E models underestimate and the ST2 model overestimates $T_{\mathrm{MD}}$, it may be possible to construct a good nonpolarizable potential with the correct $T_{\mathrm{MD}}$. Recently, a five-site potential, TIP5P, which combines features of both the TIP4P and ST2 models, has been developed to give a density maximum near $277 \mathrm{~K} .{ }^{119}$ Therefore, polarizability is not necessary to get an accurate $T_{\mathrm{MD}}$, but the polarizable models have not been fitted to reproduce this property. ${ }^{24,28,65}$

The value of the $T_{\mathrm{MD}}$ is closely linked to the value of the melting temperature. For both the water models SPC/E and TIP4P and the water isotopes, the higher the $T_{\mathrm{MD}}$ the higher the melting temperature. The melting temperature for TIP4P-FQ water has not been determined but the results presented here indicate that over a 4 ns simulation the ice phase was stable, in contrast to simulations of SPC/E water at 260 $\mathrm{K}$ (above the melting temperature of SPC/E water) in which the solid began to melt after 220 ps. ${ }^{68}$ In addition, the solid remains in a proton-disordered crystal without defects, unlike simulations with SPC/E or TIP4P. ${ }^{68,69}$ The dipole and quadrupole moments of the solid are in close agreement with the induction model estimate. ${ }^{42}$ The TIP4P-FQ model, therefore, has an accurate dipole moment in three phases: liquid, gas, and ice Ih. The dipole moment increases with decreasing temperature for the liquid and increases by about $0.4 \mathrm{D}$ going from the liquid to the solid. In addition, the width of the dipole moment distribution decreases in the solid phase, indicating that the solid is a more homogeneous electrostatic environment than the liquid.

For other solid properties, the TIP4P-FQ model is not as accurate. The density is too high and the heat of sublimation, $\Delta H_{\text {sub }}$, is too large relative to experimental values, which suggests that the potential is too attractive. The ice densities using TIP4P and SPC/E are also higher than the experimental values. ${ }^{68,69,103} \mathrm{~A}$ small increase in the Lennard-Jones $\sigma$ to give the correct solid density leads to an improved value of
$\Delta H_{\text {sub }}$ as well. Studies of gas-liquid coexistence find that decreasing $\sigma$ led to improvements in the coexistence curve. ${ }^{73}$ This, together with the underestimated value of the isothermal compressibility (Fig. 6), suggests that the Lennard-Jones form might be too sharply repulsive to be applicable to a wide variety of phases and that other functional forms may be better. Alternatively, as suggested by electronic structure calculations, the size of the molecule depends on the charge distribution. ${ }^{120}$ For the solid phase, with bigger charges, the size of the molecule should be larger and for the high temperature fluid phases, with smaller charges, the size should be smaller. One method for coupling the Lennard-Jones parameters to the charge values has recently been introduced. ${ }^{28}$ Significant progress is being made in the understanding of molecular interactions leading to better potential models, but more work needs to be done and a water model has not been demonstrated that works well for all three phases. It seems clear at this point that the addition of polarizability does lead to an improved description of water.

\section{ACKNOWLEDGMENTS}

This project has been funded in whole or in part with Federal funds from the National Cancer Institute, National Institutes of Health, under Contract No. NO1-CO-56000.

\footnotetext{
${ }^{1}$ A. Wallqvist and R. D. Mountain, in Reviews in Computational Chemistry, edited by K. B. Lipkowitz and D. B. Boyd (Wiley, New York, 1999), pp. 183-247.

${ }^{2}$ K. Laasonen, M. Sprik, M. Parrinello, and R. Car, J. Chem. Phys. 99, 9080 (1993).

${ }^{3}$ P. L. Silvestrelli and M. Parrinello, J. Chem. Phys. 111, 3572 (1999).

${ }^{4}$ L. Delle Site, A. Alavi, and R. M. Lynden-Bell, Mol. Phys. 96, 1683 (1999).

${ }^{5}$ F. H. Stillinger and C. W. David, J. Chem. Phys. 69, 1473 (1978).

${ }^{6}$ P. Barnes, J. L. Finney, J. D. Nicholas, and J. E. Quinn, Nature (London) 282, 459 (1979).

${ }^{7}$ T. P. Lybrand and P. A. Kollman, J. Chem. Phys. 83, 2923 (1985).

${ }^{8}$ J. A. C. Rullman and P. T. van Duijnen, Mol. Phys. 63, 451 (1988).

${ }^{9}$ M. Sprik and M. L. Klein, J. Chem. Phys. 89, 7556 (1988).

${ }^{10}$ P. Ahlström, A. Wallqvist, S. Engström, and B. Jönsson, Mol. Phys. 68, 563 (1989).

${ }^{11}$ P. Cieplak, P. Kollman, and T. Lybrand, J. Chem. Phys. 92, 6755 (1990).

${ }^{12}$ S. Kuwajima and A. Warshel, J. Phys. Chem. 94, 960 (1990)

${ }^{13}$ L. X. Dang, J. Chem. Phys. 97, 2659 (1992).

${ }^{14}$ R. E. Kozack and P. C. Jordan, J. Chem. Phys. 96, 3120 (1992).

${ }^{15}$ A. Wallqvist and B. J. Berne, J. Phys. Chem. 97, 13841 (1993).

${ }^{16}$ D. N. Bernardo, Y. Ding, K. Krogh-Jespersen, and R. M. Levy, J. Phys. Chem. 98, 4180 (1994).

${ }^{17}$ J. W. Caldwell and P. A. Kollman, J. Phys. Chem. 99, 6208 (1995).

${ }^{18}$ J. Blodholt, M. Sampoli, and R. Vallauri, Mol. Phys. 86, 149 (1996).

${ }^{19}$ A. A. Chialvo and P. T. Cummings, J. Chem. Phys. 105, 8274 (1996).

${ }^{20}$ S.-B. Zhu, S. Singh, and G. W. Robinson, J. Chem. Phys. 95, 2791 (1991).

${ }^{21}$ S. W. Rick, S. J. Stuart, and B. J. Berne, J. Chem. Phys. 101, 6141 (1994).

${ }^{22}$ S. W. Rick and B. J. Berne, J. Am. Chem. Soc. 118, 672 (1996).

${ }^{23}$ B.-C. Perng, M. D. Newton, F. O. Raineri, and H. L. Friedman, J. Chem. Phys. 104, 7153 (1996).

${ }^{24}$ I. M. Svishchev, P. G. Kusalik, P. G. Wang, and R. J. Boyd, J. Chem. Phys. 105, 4742 (1996).

${ }^{25}$ M. J. Field, Mol. Phys. 91, 835 (1997).

${ }^{26}$ Y.-P. Liu, K. Kim, B. J. Berne, R. A. Friesner, and S. W. Rick, J. Chem. Phys. 108, 4739 (1998).

${ }^{27}$ J. L. Banks, G. A. Kaminski, R. Zhou, D. T. Mainz, B. J. Berne, and R. A. Friesner, J. Chem. Phys. 110, 741 (1999).

${ }^{28}$ B. Chen, J. Xing, and J. I. Siepmann, J. Phys. Chem. B 104, 2391 (2000).

${ }^{29}$ S. W. Rick and R. E. Cachau, J. Chem. Phys. 112, 5230 (2000).

${ }^{30}$ J.-C. Soetens and C. Millot, Chem. Phys. Lett. 235, 22 (1995).
} 
${ }^{31}$ H. A. Stern, G. A. Kaminski, J. L. Banks, R. Zhou, B. J. Berne, and R. A. Friesner, J. Phys. Chem. B 103, 4730 (1999).

${ }^{32}$ W. B. Bosma, L. E. Fried, and S. Mukamel, J. Chem. Phys. 98, 4413 (1992).

${ }^{33}$ S. Saito and I. Ohmine, J. Chem. Phys. 106, 4889 (1997).

${ }^{34}$ B. D. Bursulaya and H. K. Kim, J. Phys. Chem. B 101, 10994 (1997).

${ }^{35}$ P.-O. Åstrand, K. Ruud, K. V. Mikkelsen, and T. Helgaker, J. Phys. Chem. A 102, 7686 (1998).

${ }^{36}$ K. Kitaura and K. Morokuma, Int. J. Quantum Chem. 10, 325 (1976).

${ }^{37}$ F. Weinhold, J. Mol. Struct. 399, 181 (1997).

${ }^{38}$ A. van der Vaart and K. M. Mertz, Jr., J. Am. Chem. Soc. 121, 9182 (1999).

${ }^{39}$ J. Korchowiec and T. Uchimaru, J. Chem. Phys. 112, 1623 (2000).

${ }^{40}$ E. D. Isaacs, A. Shukla, P. M. Platzman, D. R. Hamann, B. Barbiellini, and C. A. Tulk, Phys. Rev. Lett. 82, 600 (1999).

${ }^{41}$ T. K. Ghanty, V. N. Staroverov, P. K. Koren, and E. R. Davidson, J. Am. Chem. Soc. 122, 1210 (2000).

${ }^{42}$ E. R. Batista, S. S. Xantheas, and H. Jónsson, J. Chem. Phys. 109, 4546 (1998).

${ }^{43}$ C. Coulson and D. Eisenberg, Proc. R. Soc. London, Ser. A 291, 445 (1966).

${ }^{44}$ E. R. Batista, S. S. Xantheas, and H. Jónsson, J. Chem. Phys. 111, 6011 (1999).

${ }^{45}$ E. R. Batista, S. S. Xantheas, and H. Jónsson, J. Chem. Phys. 112, 3285 (2000).

${ }^{46}$ S. L. Carnie and G. N. Patey, Mol. Phys. 47, 1129 (1982).

${ }^{47}$ M. Sprik, J. Chem. Phys. 95, 6762 (1991).

${ }^{48}$ K. Watanabe and M. L. Klein, Chem. Phys. 131, 157 (1989).

${ }^{49}$ M. Sprik, J. Phys. Chem. 95, 2283 (1991).

${ }^{50}$ M. N. Afar and J. B. Hasted, J. Opt. Soc. Am. 67, 902 (1977).

${ }^{51}$ S. Krishnamurthy, R. Bansil, and J. Wiafe-Akenten, J. Chem. Phys. 79, 5863 (1983).

${ }^{52}$ P. A. Madden and R. W. Impey, Chem. Phys. Lett. 123, 502 (1986).

${ }^{53}$ R. Frattini, M. Sampoli, M. A. Ricci, and G. Ruocco, Chem. Phys. Lett. 141, 297 (1987).

${ }^{54}$ B. Guillot, J. Chem. Phys. 95, 1543 (1991).

${ }^{55}$ G. E. Walrafen, in Water-A Comprehensive Treatise, edited by F. Franks (Plenum, New York, 1972).

${ }^{56}$ R. H. Wood, E. M. Yezdimer, S. Sakane, J. A. Barriocanal, and D. J. Doren, J. Chem. Phys. 110, 1329 (1999).

${ }^{57}$ F. H. Stillinger and A. Rahman, J. Chem. Phys. 60, 1545 (1974).

${ }^{58}$ P. H. Poole, F. Sciortino, U. Essman, and H. E. Stanley, Nature (London) 360, 324 (1992).

${ }^{59}$ S. R. Billeter, P. M. King, and W. F. van Gunsteren, J. Chem. Phys. 100, 6692 (1994).

${ }^{60}$ L. A. Báez and P. Clancy, J. Chem. Phys. 101, 9837 (1994).

${ }^{61}$ A. Wallqvist and P.-O. Astrand, J. Chem. Phys. 102, 6559 (1995).

${ }^{62}$ S. Harrington, P. H. Poole, F. Sciortino, and H. E. Stanley, J. Chem. Phys. 107, 7443 (1997).

${ }^{63}$ K. Bagchi, S. Balasubramanian, and M. L. Klein, J. Chem. Phys. 107, 8561 (1997).

${ }^{64}$ W. L. Jorgensen and C. Jensen, J. Comput. Chem. 19, 1179 (1998).

${ }^{65}$ P. Jedlovszky and R. Vallauri, Mol. Phys. 97, 1157 (1999).

${ }^{66}$ W. L. Jorgensen, J. Chandrasekhar, J. D. Madura, R. W. Impey, and M. L. Klein, J. Chem. Phys. 79, 926 (1983).

${ }^{67}$ H. J. C. B. J. R. Grigera and T. P. Straatsma, J. Phys. Chem. 91, 6269 (1987).

${ }^{68}$ L. A. Báez and P. Clancy, Mol. Phys. 86, 385 (1995).

${ }^{69}$ M. J. Vlot, J. Huinink, and J. P. van der Eerden, J. Chem. Phys. 110, 55 (1999).

${ }^{70} \mathrm{~S}$. W. Rick, in Simulation and Theory of Electrostatic Interactions in Solution, edited by L. R. Pratt and G. Hummer (American Institute of Physics, Melville, NY, 1999), pp. 114-126.

${ }^{71}$ T. M. Nymand and P. Linse, J. Chem. Phys. 112, 6386 (2000).

${ }^{72}$ J. Brodholt, M. Sampoli, and R. Vallauri, Mol. Phys. 85, 81 (1995).

${ }^{73}$ K. Kiyohara, K. E. Gubbins, and A. Z. Panagiotopoulos, Mol. Phys. 94, 803 (1998).

${ }^{74}$ E. M. Yezdimer and P. T. Cummings, Mol. Phys. 97, 993 (1999).
${ }^{75}$ I. M. Svishchev and T. M. Hayward, J. Chem. Phys. 111, 9034 (1999).

${ }^{76}$ B. Chen, J. J. Potoff, and J. I. Siepmann, J. Phys. Chem. B 104, 2378 (2000).

${ }^{77}$ J. M. Sorenson, G. Hura, A. K. Soper, A. Pertsemlidis, and T. HeadGordon, J. Phys. Chem. B 103, 5413 (1999).

${ }^{78}$ S. J. Stuart and B. J. Berne, J. Phys. Chem. 100, 11934 (1996).

${ }^{79}$ S. W. Rick and B. J. Berne, J. Phys. Chem. B 101, 10488 (1997).

${ }^{80}$ J. S. Bader and B. J. Berne, J. Chem. Phys. 104, 1293 (1996).

${ }^{81}$ E. Stöckelmann and R. Hentschke, J. Chem. Phys. 110, 12097 (1999).

${ }^{82}$ M. Medeiros and M. E. Costas, J. Chem. Phys. 107, 2012 (1997).

${ }^{83}$ R. A. Bryce, M. A. Vincent, and I. A. Hillier, J. Phys. Chem. A 103, 4094 (1999).

${ }^{84}$ M. D. Elola, D. A. Estrin, and D. Laria, J. Phys. Chem. A 103, 5105 (1999).

${ }^{85}$ R. G. Parr and W. Yang, Density-Functional Theory of Atoms and Molecules (Oxford University Press, Oxford, 1989).

${ }^{86}$ R. T. Sanderson, Science 114, 670 (1951).

${ }^{87}$ L. Došen-Mićović, D. Jeremić, and N. L. Allinger, J. Am. Chem. Soc. 105, 1716 (1983).

${ }^{88}$ W. J. Mortier, K. V. Genechten, and J. Gasteiger, J. Am. Chem. Soc. 107, 829 (1985).

${ }^{89}$ K. T. No, J. A. Grant, and H. A. Scheraga, J. Phys. Chem. 94, 4732 (1990).

${ }^{90}$ A. K. Rappé and W. A. Goddard, J. Phys. Chem. 95, 3358 (1991).

${ }^{91}$ C. A. Reynolds, J. W. Essex, and W. G. Richards, J. Am. Chem. Soc. 114, 9075 (1993)

${ }^{92}$ D. van Belle, M. F. G. Lippens, and S. J. Wodak, Mol. Phys. 77, 239 (1992).

${ }^{93}$ H. C. Andersen, J. Chem. Phys. 72, 2384 (1980).

${ }^{94}$ G. Ciccotti and J. P. Ryckaert, Comput. Phys. Rep. 4, 345 (1986).

${ }^{95}$ G. J. Martyna, D. J. Tobias, and M. L. Klein, J. Chem. Phys. 101, 4177 (1994).

${ }^{96}$ S. Nosé, Mol. Phys. 52, 255 (1984).

${ }^{97}$ W. G. Hoover, Phys. Rev. A 31, 1695 (1985).

${ }^{98}$ M. Parrinello and A. Rahman, Phys. Rev. Lett. 45, 1196 (1980).

${ }^{99}$ J. A. Hayward and J. R. Reimers, J. Chem. Phys. 106, 1518 (1997).

${ }^{100}$ M. P. Allen and D. J. Tildesley, Computer Simulation of Liquids (Oxford University Press, Oxford, 1987).

${ }^{101}$ G. S. Kell, J. Chem. Eng. Data 20, 97 (1975).

${ }^{102}$ D. Eisenberg and W. Kauzmann, The Structure and Properties of Water (Oxford University Press, New York and Oxford, 1969).

${ }^{103}$ O. A. Karim and A. D. J. Haymet, J. Chem. Phys. 89, 6889 (1988).

${ }^{104}$ T. Dyke and J. Muenter, J. Chem. Phys. 59, 3125 (1973).

${ }^{105}$ J. Verhoeven and A. Dymanus, J. Chem. Phys. 52, 3222 (1970).

${ }^{106}$ U. Kaatze and V. Uhlendorf, Z. Phys. Chem., Neue Folge 126, 151 (1981).

${ }^{107}$ CRC Handbook of Chemistry and Physics, edited by R. C. Weast (CRC Press, Boca Raton, 1985-1986), Vol. 66.

${ }^{108}$ M. Rami Ready and M. Berkowitz, Chem. Phys. Lett. 155, 173 (1989).

${ }^{109}$ H. E. Alper and R. M. Levy, J. Chem. Phys. 91, 1242 (1989).

${ }^{110}$ Y. Guissani and B. Guillot, J. Chem. Phys. 98, 8221 (1993).

${ }^{111}$ R. Frattini, M. A. Ricci, G. Ruocco, and M. Sampoli, J. Chem. Phys. 92, 2540 (1990).

${ }^{112}$ H. R. Pruppacher, J. Chem. Phys. 56, 101 (1983).

${ }^{113}$ F. X. Prielmeier, E. W. Lang, R. J. Speedy, and H.-D. Lüdemann, Phys. Rev. Lett. 59, 1128 (1987).

${ }^{114}$ R. J. Speedy and C. A. Angell, J. Chem. Phys. 65, 851 (1976).

${ }^{115}$ F. W. Starr, S. Harrington, F. Sciortino, and H. E. Stanley, Phys. Rev. Lett. 82, 3629 (1999).

${ }^{116}$ J. Jonas, T. DeFries, and D. J. Wilbur, J. Chem. Phys. 65, 582 (1976).

${ }^{117}$ N. E. Dorsey, Properties of Ordinary Water-Substance in all its Phases: Water Vapor, Water, and all the Ices (Reinhold, New York, 1940).

${ }^{118}$ E. Whalley, in Physics and Chemistry of Ice, edited by E. Whalley, S. J. Jones, and L. W. Gold (Royal Society of Canada, Ottawa, 1993).

${ }^{119}$ M. W. Mahoney and W. L. Jorgensen, J. Chem. Phys. 112, 8910 (2000).

${ }^{120}$ J. K. Badenhoop and F. Weinhold, J. Chem. Phys. 107, 5422 (1997).

${ }^{121}$ M. Neumann, J. Chem. Phys. 85, 1567 (1986). 